\title{
ANÁLISIS DEL PROGRAMA DE SEGUIMIENTO DE MEDIDAS JUDICIALES DE RÉGIMEN ABIERTO EN LA CIUDAD DE ALICANTE DURANTE 1.996
}

\author{
JAVIER DOMÍNGUEZ \\ JOSÉ R. BROCAL \\ JOSÉ J. CASES \\ VIRGINIA TOVAR \\ AGUSTÍN BUENO
}

\section{RESUMEN}

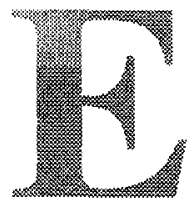

n este trabajo se presentan los datos correspondientes a la problemática presentada en los casos de menores con aplicación de medidas judiciales en medio abierto en la ciudad de Alicante en 1996 y la intervención efectuada en los mismos: características de población, medidas judiciales empleadas y distribución de las mismas por delitos, sexo y edad, objetivos según las medidas, recursos utilizados y niveles de logro apreciados. Se incluye una proyección del programa hacia el futuro.

\section{PALABRAS CLAVE}

Jóvenes infractores, medidas judiciales, intervención social, medio abierto.

I. AMBIENTACIÓN DEL PROGRAMA DE SEGUIMIENTO DE MEDIDAS JUDICIALES DE RÉGIMEN ABIERTO EN LA CIUDAD DE ALICANTE.

Consideramos necesario contextualizar el Programa de Seguimiento de las Medidas Judiciales de Régimen Abierto antes de entrar en el

Este programa se realiza dentro del convenio de colaboración entre Nazaret y el Excmo. Ayuntamiento de Alicante. 
análisis de los datos correspondientes al año 1996. Pretendemos con ello darles el alcance que se les puede pedir, que es el de primerizos, aunque herederos de una larga historia de hacer sin hacer, o de estar en la Ley pero no en la realidad social. Podemos afirmar sin reparos que 1996 es el primer año natural completo en el que hay seguimiento real de una gama de medidas judiciales de régimen abierto en la ciudad de Alicante.

En la antigua Ley de Tribunales Tutelares de Menores de 1948 se establecían como posibles medidas a ser tomadas por el juez respecto a un menor de edad: el internamiento en un centro y la libertad vigilada. A ésta se reducían, pues, las medidas de régimen abierto, y de hecho no significaba intervención ni ejecución salvo de carácter administrativo.

En aquel contexto legal, que ha durado hasta hace muy poco, el menor era llamado al Tribunal Tutelar de Menores y allí recibía la amonestación correspondiente y se le informaba que se le había abierto un expediente. Ahí quedaba todo, el expediente en su archivo y el menor haciendo la misma vida que antes. Si volvía a delinquir y era cogido por la policía entonces se repetía la misma función. Así, hasta que ingresaba en un centro o superaba la edad penal y pasaba a la jurisdicción ordinaria. Para informar sobre los expedientes de libertad vigilada el Ministerio de Justicia tenía en cada provincia entre dos y cuatro delegados, que emitían sus informes si el juez se lo requería -y que si los hubiera requerido en todos los casos, no habría sido posible materialmente realizarlos-.

El 5 de junio de 1992 es aprobada la Ley 4/92, sobre reforma de la Ley Reguladora de la Competencia y Procedimiento de los Juzgados de Menores. Esta ley, promulgada por inconstitucionalidad de la de 1948, modifica importantes artículos de la anterior. En concreto, el Artículo Segundo, apartado cuatro, dice así:

"El artículo 17 de la Ley de Tribunales Tutelares de Menores, aprobada por Decreto de 11 de junio de 1948, tendrá la siguiente redacción:

El Juez de menores podrá acordar, con respecto a éstos, las medidas siguientes:

1. a Amonestación o internamiento por tiempo de uno a tres fines de semana.

2. ${ }^{2}$ Libertad vigilada.

3. Acogimiento por otra persona o núcleo familiar.

4. ${ }^{a}$ Privación del derecho a conducir ciclomotores o vehículos de motor.

5. ${ }^{2}$ Prestación de servicios en beneficio de la Comunidad. 


\section{6. ${ }^{a}$ Tratamiento ambulatorio o ingreso en un Centro de carácter terapéutico.}

7. Ingreso en un Centro en régimen abierto, semiabierto o cerrado.

En el ejercicio de la facultad de enjuiciar las faltas a que se refiere el apartado $2^{\circ}$ del artículo 9 se aplicarán las penas señaladas en el Código Penal". ${ }^{2}$

Así mismo, establece un nuevo procedimiento de actuación de los juzgados de menores, con informe del fiscal, asesorado por un equipo técnico, y se concreta que "la ejecución de las medidas adoptadas por los Juzgados de Menores corresponden a las Entidades Públicas competentes en la materia" (disposición adicional tercera de la citada Ley 4/92). La Entidad Pública, en nuestro caso, es la Comunidad Autónoma, que delega esta función en los Ayuntamientos.

El Ayuntamiento de Alicante concierta con la Fundación Nazaret -P. Fontova- la ejecución del Programa de Seguimiento de Medidas Judiciales de Régimen Abierto. Esto se produce en el curso escolar 93/94, incluye un único profesional (los delegados del Ministerio de Justicia habían desaparecido) y alcanza solamente a la medida de libertad vigilada porque las otras no están todavía diseñadas en la Comunidad Valenciana.

En este momento, cabía un modelo burocrático y de control del menor, o un modelo educativo con intención de incidir positivamente en el proceso socializador del menor. Este segundo modelo era el que se planteaba desde la dirección del programa, aunque fuera con los mínimos recursos materiales y personales que se ofrecían. Curiosamente, coincide la puesta en marcha del programa con una larga vacante del titular del Juzgado de Menores en la provincia de Alicante.

El 5 de diciembre de 1994 es aprobada por las Cortes Valencianas la Ley 7/94 de la Infancia ${ }^{3}$ en la cual se concretizan, dentro del capítulo de programas de reinserción, las medidas judiciales de todo tipo que puede imponer el juez a los menores (Cfr. Artículo 29 y ss. de la Ley $7 / 94$ de la Generalitat Valenciana) y cuya ejecución es competencia de la Generalitat ${ }^{4}$.

Completado así el marco legal, aunque sin ninguna otra infraestructura administrativa o de especial capacidad de gestión de recursos,

Cfr. BOE, $\mathrm{N}^{\circ}$ 140, de 11 de junio de 1992.

Cfr. DOGV, $\mathrm{N}^{\circ} 2.408$ de 16 de diciembre de 1994.

4 Un desarrollo del diseño de esos programas de reinserción puede consultarse en: MOYA, C,; GALVAÑ. F. y NIETO, C.: "Programas de ejecución de medidas judiciales". En A. BUENO (Coord.): Intervención social con menores. Alicante, Secretariado de Publicaciones de la Universidad de Alicante, 1996, pp. 293 - 318. 1: Cfr. BOE, $N^{\circ}$ 140, de 11 de junio de 1992. 
a comienzos del curso 1995/96 se amplía el concierto del Ayuntamiento de Alicante a tres educadores (titulados en Trabajo Social, Psicología y Profesorado de EGB, respectivamente) y a toda la gama de medidas judiciales de régimen abierto que de hecho pone en ejecución la Comunidad Valenciana, que son: libertad vigilada, internamiento de fin de semana, prestación de servicios en beneficio de la comunidad.

Todo este largo proceso legislativo-administrativo es lo que explica que el año 1996 sea el primer año natural completo que permite un análisis del programa. Es el primer año completo, aunque tiene un largo precedente bastante irregular, por lo cual el análisis no permite todavía comparaciones retrospectivas ni prospecciones de evolución.

\section{SITUACIÓN GENERAL DEL RECURSO}

- Número de medidas: 76.

- Número de menores: 73.

- Menores con más de una medida: 3 (tres menores con dos medidas cada uno). Uno de ellos con post-medida, al no haber sido conseguidos los objetivos planteados durante la medida, y con total voluntariedad por parte del menor y su familia.

- Menores que han causado baja durante 1996: 49.

- Medidas que han causado baja durante 1996: 50.

- Medidas iniciadas en 1996: 53.

\section{III.ESTUDIO DE VARIABLES DE LA POBLACIÓN ATENDIDA}

3.1. Distribución de menores por sexo $(n=73)$.

\begin{tabular}{|c|c|c|c|c|}
\hline CHICOS & $\%$ & CHICAS & $\%$ & TOTAL \\
\hline 63 & 86,3 & 10 & 13,7 & 73 \\
\hline
\end{tabular}

TABLA 1

Como podemos observar, el grupo de hombres con medida judicial durante el año 1996 es significativamente mayor que el grupo de mujeres. Por cada chica con medidas judiciales de régimen abierto hay seis varones en la misma consideración.

\subsection{Distribución de menores por año de nacimiento y sexo $(n=73)$.}

El rango de edad donde se sitúan la mayoría de los casos se encuentra entre los años de nacimiento 1980 y $1982(82,2 \%)$, entre 14 y 


\begin{tabular}{|c|c|c|c|c|c|c|}
\hline \multirow[b]{2}{*}{ AÑO NACIM. } & \multicolumn{4}{|c|}{ SEXO } & \multirow[b]{2}{*}{ TOTAL } & \multirow[b]{2}{*}{$\%$} \\
\hline & CHICOS & $\%$ & CHICAS & $\%$ & & \\
\hline Año 78 & 1 & 1,6 & 1 & 10 & 2 & 2,7 \\
\hline Año 79 & 9 & 14,3 & 0 & 0 & 9 & 12,3 \\
\hline Año 80 & 28 & 44,4 & 3 & 30 & 31 & 42,5 \\
\hline Año 81 & 13 & 20,6 & 2 & 20 & 15 & 20,5 \\
\hline Año 82 & 10 & 15,9 & 4 & 40 & 14 & 19,2 \\
\hline Año 83 & 2 & 3,2 & 0 & 0 & 2 & 2,7 \\
\hline TOTAL & 63 & 100 & 10 & 100 & 73 & 100 \\
\hline
\end{tabular}

TABLA 2

16 años. En este grupo destaca el grupo de hombres nacidos durante 1980, frente al grupo de mujeres, más numeroso, nacidas durante 1982. El hecho de que el grupo de hombres tenga, en conjunto y como promedio, más edad que el grupo de mujeres puede apuntar a la mayor precocidad madurativa de las chicas, si bien el volumen de infracciones judicializadas o sancionadas son sensiblemente menores.

Los casos de edades superiores a los 16 años (15\%) corresponden a delitos que se cometieron cuando los menores no habían cumplido aún esa edad.

El número de menores que poseen menos de 14 (2,7\%) es muy bajo y son en su totalidad hombres. Este dato tanto nos puede informar de la edad paradigmática del comienzo general de la actividad delictiva, como la consideración social -tanto formal como informal- de la misma.

3.3. Distribución de medidas por año y trimestre del hecho cometido, resolución e inicio de medida $(n=76)$

En la tabla 3 se presentan los datos unificados y ordenados según el año y trimestre del hecho cometido que originó la medida judicial impuesta, la resolución (decisión judicial o sentencia) y el inicio de la medida (firma del menor y padres, así como el primer esbozo del plan de trabajo de la medida).

\subsubsection{Trimestre y año del hecho cometido}

De las medidas ejecutadas durante el año 1996, con respecto a la fecha del delito cometido, (véase tabla 4) el mayor porcentaje corresponde a hechos que se cometieron durante el año 95 y $96(82,9 \%)$, siendo el $17,1 \%$ restante a hechos que se cometieron anteriores al 95 . Se puede observar un mayor número de delitos cometidos durante el $2^{\circ}, 3^{\circ}$ y $4^{\circ}$ trimestre del 95 . 


\begin{tabular}{|c|c|c|c|c|c|c|c|}
\hline Año & Trimestre & Hecho cometido & $\%$ & Resolución & $\%$ & Inicio & $\%$ \\
\hline 1993 & 1 & 1 & 1,3 & 0 & 0 & 0 & 0 \\
\hline & 2 & 1 & 1,3 & 0 & 0 & 0 & 0 \\
\hline & 3 & 0 & 0 & 0 & 0 & 0 & 0 \\
\hline & 4 & 1 & 1,3 & 0 & 0 & 0 & 0 \\
\hline \multicolumn{2}{|c|}{ TOTAL 1993 } & 3 & 3,9 & 0 & 0 & 0 & 0 \\
\hline 1994 & 1 & 2 & 2,6 & 0 & 0 & 0 & 0 \\
\hline & 2 & 4 & 5,3 & 0 & 0 & 0 & 0 \\
\hline & 3 & 2 & 2,6 & 1 & 1,3 & 1 & 1,3 \\
\hline & 4 & 2 & 2,6 & 5 & 6,6 & 1 & 1,3 \\
\hline TOTAL 1994 & 10 & 13,2 & 6 & 7,9 & 2 & 2,6 \\
\hline 1995 & 1 & 4 & 5,3 & 5 & 6,6 & 0 & 0 \\
\hline & 2 & 21 & 27,6 & 5 & 6,6 & 4 & 5,3 \\
\hline & 3 & 11 & 14,5 & 2 & 2,6 & 1 & 1,3 \\
\hline & 4 & 12 & 15,8 & 14 & 18,4 & 16 & 21,1 \\
\hline \multicolumn{2}{|l|}{ TOTAL 1995 } & 48 & 63,2 & 26 & 34,2 & 21 & 27,6 \\
\hline 1996 & 1 & 9 & 11,8 & 20 & 26,3 & 15 & 19,7 \\
\hline & 2 & 5 & 6,6 & 12 & 15,8 & 24 & 31,6 \\
\hline & 3 & 1 & 1,3 & 3 & 3,9 & 3 & 3,9 \\
\hline & 4 & 0 & 0 & 9 & 11,8 & 11 & 14,5 \\
\hline
\end{tabular}

TABLA 3

\begin{tabular}{|l|c|c|c|c|}
\hline Año & ${\text { Trimestre } 1 .^{\circ}}^{\circ}$ & ${\text { Trimestre } 2 .^{\circ}}^{\circ}$ & ${\text { Trimestre } 3 .^{\circ}}^{\circ}$ & ${\text { Trimestre } 4 .^{\circ}}^{\circ}$ \\
\hline 93 & 1 & 1 & 0 & 1 \\
\hline 94 & 2 & 4 & 2 & 2 \\
\hline 95 & 4 & 21 & 11 & 12 \\
\hline 96 & 9 & 5 & 1 & 0 \\
\hline TOTAL & 16 & 31 & 14 & 15 \\
\hline
\end{tabular}

TABLA 4

Si miramos estos datos de año y trimestre $(n=76)$, relativos al hecho cometido en agrupaciones trimestrales, obtenemos la tabla 4 de distribución de los casos.

Hay una mayor incidencia acumulativa de hechos cometidos durante los segundos trimestres del año - meses de abril, mayo y junio-. Es un dato sugerente pero que necesitará mayor comprobación, sobre todo teniendo en cuenta que son los años $94 \mathrm{y}$, sobre todo, 95 los que marcan esta tendencia. El año 93 no facilita datos valorativos, y el año 96 manifiesta una tendencia distinta 


\subsubsection{Trimestre y año de la resolución}

De las medidas ejecutadas durante este periodo, más de la mitad $(57,9 \%)$ corresponden a resoluciones emitidas durante 1996, destacando el $1^{\mathrm{er}}$ y $2^{\circ}$ trimestre del mismo. También resalta el número de resoluciones emitidas durante el $4^{\circ}$ trimestre de 1995 . No existe ninguna resolución anterior al $2^{\circ}$ semestre de 1994 , lo cual se debe al hecho, comentado en la ambientación (epígrafe 1), de que el primer año del programa comienza con un periodo del Juzgado de Menores de la Provincia de Alicante sin Juez en ejercicio.

\subsubsection{Trimestre y año de inicio de medidas}

Durante 1996 se han iniciado 53 medidas $(69,7 \%)$ de las medidas ejecutadas durante este periodo, destacando el $1^{\text {er }}$ semestre y el $4^{\circ}$ trimestre del mismo, habiendo un descenso significativo durante el $3 \mathrm{er}$. trimestre. También resalta el número de medidas iniciadas en el $4^{\circ}$ trimestre del año 1995. No se ejecutaron medidas judiciales anteriores al $2^{\circ}$ semestre de 1994, por la misma razón de juzgado vacante que hemos indicado antes. Al inicio del año evaluado 23 medidas $(30,2 \%)$ habían sido iniciadas con anterioridad a 1996.

\subsection{Relación de medidas respecto al tiempo transcurrido (en meses)} entre el hecho cometido y la resolución $(n=96)$

\begin{tabular}{|c|c|c|}
\hline Tiempo transcurrido en meses & TOTAL & $\%$ \\
\hline 1 & 1 & 1,3 \\
\hline 2 & 2 & 2,6 \\
\hline 3 & 2 & 2,6 \\
\hline 4 & 12 & 15,8 \\
\hline 5 & 17 & 22,4 \\
\hline 6 & 12 & 15,8 \\
\hline 7 & 7 & 9,2 \\
\hline 8 & 4 & 5,3 \\
\hline 9 & 8 & 10,5 \\
\hline 10 & 2 & 2,6 \\
\hline 11 & 1 & 1,3 \\
\hline 12 & 4 & 5,3 \\
\hline 14 & 2 & 2,6 \\
\hline 20 & 1 & 1,3 \\
\hline 21 & 1 & 1,3 \\
\hline TOTAL & 76 & 100 \\
\hline
\end{tabular}

TABLA 5 (tiempo medio -en meses-: 6,41) 
Del total de medidas ejecutadas durante este periodo, podemos observar que el tiempo medio de demora entre el hecho cometido y la resolución oscila en la mitad de las medidas (54\%) entre 4 y 6 meses, siendo un $6,5 \%$ de las medidas las que poseen una menor demora. En el resto de medidas $(39,5 \%)$ es superior a 6 meses, y aquéllas que superan el año constituyen el 5,2\%.

Estos datos son importantes de resaltar, puesto que al tratar con menores es de vital trascendencia que el tiempo entre hecho cometido y resolución sea lo más breve posible, para que la medida sea realmente educativa y el menor pueda establecer con claridad la conexión entre hecho delictivo y sus consecuencias.

\begin{tabular}{|c|c|c|c|}
\hline MEDIDA & DURACIÓN & TOTAL & $\%$ \\
\hline \multirow[t]{2}{*}{ Fines de semana } & 2 & 2 & 2,6 \\
\hline & 3 & 5 & 6,6 \\
\hline \multicolumn{2}{|l|}{ TOTAL iines de semana } & 7 & 9,2 \\
\hline \multirow[t]{12}{*}{ Libertad vigilada } & 1 semana & 1 & 1,3 \\
\hline & curso escolar & 7 & 9,2 \\
\hline & 2 meses & 2 & 2,6 \\
\hline & 3 meses & 2 & 2,6 \\
\hline & 4 meses & 2 & 2,6 \\
\hline & 6 meses & 13 & 17,1 \\
\hline & 8 meses & 3 & 3,9 \\
\hline & 9 meses & 6 & 7,9 \\
\hline & 1 año & 16 & 21,1 \\
\hline & 2 años & 1 & 1,3 \\
\hline & hasta 16 años & 1 & 1,3 \\
\hline & hasta 18 años & 1 & 1,3 \\
\hline \multicolumn{2}{|l|}{ TOTAL libertad vigilada } & 56 & 73,7 \\
\hline \multirow[t]{4}{*}{ Prestación servicios comunidad } & 10 horas & 1 & 1,3 \\
\hline & 15 horas & 4 & 5,3 \\
\hline & 20 horas & 4 & 5,3 \\
\hline & 30 horas & 1 & 1,3 \\
\hline \multicolumn{2}{|l|}{ TOTAL prestac. serv. com. } & 10 & 13,2 \\
\hline Libertad vig. + prest. serv. com. & 1 año +30 horas & 1 & 1,3 \\
\hline \multicolumn{2}{|l|}{ TOTAL lib. vig. + prest. serv. com. } & 1 & 1,3 \\
\hline \multirow[t]{2}{*}{ Post-medida } & 1 mes & 1 & 1,3 \\
\hline & 4 meses & 1 & 1,3 \\
\hline \multicolumn{2}{|l|}{ TOTAL post-medida } & 2 & 2,6 \\
\hline
\end{tabular}




\subsection{Tipo de medidas y duración $(n=76)$.}

Hay que destacar que, de entre todas las medidas ejecutadas durante 1996 (véase tabla 6), la medida más impuesta en medio abierto es la libertad vigilada $(73,7 \%)$. En cuanto a la duración impuesta en libertad vigilada destacan los 6 meses y el año, siendo éste el periodo más adecuado, a juicio de este equipo, para poder establecer un plan de intervención y poder llevarlo a cabo con ciertas garantías, así como para poder establecer vínculos con el menor y su familia que faciliten dicha intervención. Hemos podido observar que libertades vigiladas que tienen una menor duración no son demasiado positivas por la brevedad de tiempo, y aquéllas que exceden de este tiempo se hacen difíciles de llevar, ya que se hacen muy largas en el tiempo y el chaval llega a perder la perspectiva de la medida y su relación con su conducta, llegando a plantearse el plan de intervención y perdiendo su función educativa.

Queda manifiesto que la medida de libertad vigilada en el programa actual no tiene equivalencia con la libertad vigilada ejecutada en cumplimiento de la Ley de 1948.

En cuanto a la medida de prestación de servicios en beneficio de la comunidad, es la segunda medida de medio abierto más impuesta $(13,2 \%)$. Su duración oscila entre las 10 y 30 horas. Esta medida se ha visto muy positiva en la intervención de los menores, obteniéndose muy buenos resultados por la fácil conexión entre la conducta del menor y su posterior reparación. También hay que resaltar que el perfil de chavales que tienen esta medida difiere de los de libertad vigilada.

Respecto al internamiento en domicilio durante los fines de semana, también hay que resaltar los mismos comentarios que los realizados para la prestación de servicios en beneficio de la comunidad.

Resaltar el hecho de que tan sólo en un caso una misma resolución impone dos medidas diferentes.

En cuanto a la post-medida, los casos ejecutados han sido de chavales que han tenido muy buena respuesta en medidas en ejecución y que al finalizar la medida y al comprobarse que los objetivos no han sido concluidos, y con la voluntariedad del menor y de su familia, se continúa trabajando con él.

\subsection{Distribución de medidas por tipo de delito y sexo $(n=76)$}

El delito más común es el de robo con fuerza $(27,6 \%)$ cometido únicamente por chicos (véase tabla 7). 


\begin{tabular}{|l|c|c|c|}
\hline TIPO DELITO & SEXO & TOTAL & $\%$ \\
\hline Agresión sexual & Chicos & 1 & 1,3 \\
\hline Contra salud pública & Chicos & 1 & 1,3 \\
\cline { 2 - 4 } & Chicas & 3 & 3,9 \\
\hline TOTAL contra salud pública & & 4 & 5,3 \\
\hline Falta & Chicas & 1 & 1,3 \\
\hline Falta contra el régimen de poblaciones & Chicos & 1 & 1,3 \\
\cline { 2 - 4 } & Chicas & 1 & 1,3 \\
\hline TOTAL falta contra el régimen de poblaciones & & 2 & 2,6 \\
\hline Falta de hurto & Chicos & 10 & 13,2 \\
\hline Robo con fuerza & Chicos & 21 & 27,6 \\
\hline Robo con intimidación & Chicos & 6 & 7,9 \\
\cline { 2 - 4 } & Chicas & 1 & 1,3 \\
\hline TOTAL robo con intimidación & & 7 & 9,2 \\
\hline Robo con violencia & Chicos & 7 & 9,2 \\
\cline { 2 - 4 } & Chicas & 4 & 5,3 \\
\hline TOTAL robo con violencia & & 11 & 14,5 \\
\hline Robo en grado de frustación & Chicos & 1 & 1,3 \\
\hline U.I.V.M & Chicos & 2 & 2,6 \\
\hline TOTAL U.l.V.M & Chicos & 6 & 7,9 \\
\hline Robo con intimidación + Falta de hurto & Chicas & 1 & 1,3 \\
\hline Quebrantamiento de medida & & 7 & 9,2 \\
\hline Quebrantamiento de medida + UIVM + Robo con & Chicos & 1 & 1,3 \\
\hline Receptación & Chicos & 1 & 1,3 \\
\hline Falta de hurto + Incendio + UIVM & Chicos & 2 & 1,3 \\
\hline Robo con violencia + Robo con intimidación & Chicos & 1 & 1,3 \\
\hline Post-medida & Chicos & 1 & 1,3 \\
\hline Cambio de medida & Chicos & 2 & 2,6 \\
\hline TOTAL & & 2,6 \\
\hline
\end{tabular}

TABLA 7

El segundo delito más cometido es el de robo con violencia $(14,5 \%)$. Este delito es el que más han cometido las mujeres.

La falta de hurto es el tercer delito más cometido $(13,2 \%)$ y es sólo cometido por hombres.

Siguiendo en orden descendente, la utilización ilegítima de vehículos a motor (U.I.V.M.) es el cuarto delito cometido con mayor frecuencia, siendo en casi su totalidad hombres y tan sólo una mujer; 
también en la misma proporción y con el mismo número de casos es el robo con intimidación.

En cuanto a los delitos contra la salud pública $(5,3 \%)$ destaca el grupo de mujeres frente al de hombres.

Hay que resaltar que las resoluciones que recogen varios delitos constituyen el 5,2\% de los casos y son en su totalidad hombres.

Destacar que respecto al periodo anterior (1995) los tipos de delito se han visto ampliados (casi duplicados), con la característica añadida de que, en algunos casos, para una misma resolución y un mismo menor, se corresponden varios tipos de delito.

3.7. Distribución de menores según el criterio de estructuración familiar (responsable/s educativo/s) $(n=73)$

\begin{tabular}{|l|r|r|}
\hline ESTRUCTURACIÓN FAMILIAR & TOTAL & $\%$ \\
\hline Pareja constituida de manera estable & 43 & 58,9 \\
\hline Mujer soltera habitualmente sola & 2 & 2,7 \\
\hline Mujer separada habitualmente sola & 17 & 23,3 \\
\hline Mujer viuda habitualmente sola & 2 & 2,7 \\
\hline Hombre habitualmente solo & 1 & 1,4 \\
\hline Abuelo/a & 5 & 6,8 \\
\hline Hermano/a mayor & 1 & 1,4 \\
\hline Emancipado & 2 & 2,7 \\
\hline TOTAL & 73 & 100 \\
\hline
\end{tabular}

TABLA 8

El mayor número de casos se refieren a familias estructuradas en torno a parejas estables $(58,9 \%)$. El criterio seguido para calificar una pareja como estable ha sido el de la convivencia en el mismo núcleo familiar, sin embargo, tal estabilidad, en gran número de casos, es muy relativa, debido a problemáticas individuales de cada pareja. Otro dato reseñable es el alto porcentaje de mujeres separadas, y habitualmente solas como responsable educativo.

Los dos casos de emancipación corresponden a menores que han formado su propio núcleo familiar, aunque siguen conviviendo en el domicilio de alguno de los progenitores.

\subsection{Distribución de menores por condiciones de la vivienda $(n=73)$}

Los criterios que hemos seguido para evaluar las condiciones de las viviendas (tabla 9) han sido los siguientes: 
- Condiciones precarias (falta de higiene, hacinamiento, infraestructura interna y externa insuficiente).

- Condiciones mínimas (deterioro de la infraestructura interna y externa, esto es, mobiliario inadecuado en función de la composición familiar, humedad, grietas, etc.).

- Condiciones adecuadas (infraestructura externa cuidada, mobiliario ajustado a las necesidades del núcleo familiar, higiene suficiente, ausencia de hacinamiento, etc.).

\begin{tabular}{|l|c|c|}
\hline CONDICIONES DE LA VIVIENDA & TOTAL & $\%$ \\
\hline Adecuadas & 53 & 72,6 \\
\hline Mínimas & 13 & 17,8 \\
\hline Precarias & 7 & 9,6 \\
\hline TOTAL & 73 & 100 \\
\hline
\end{tabular}

TABLA 9

3.9. Características del menor al inicio de la medida $(n=73)$

\begin{tabular}{|l|c|c|}
\hline CARACTERÍSTICAS del menor al inicio de la medida & N. ${ }^{\circ}$ CASOS & $\%$ \\
\hline Consumo de sustancias tóxicas & 23 & 31,51 \\
\hline Salud física o enfermedad importante & 1 & 1,37 \\
\hline Salud mental & 6 & 8,22 \\
\hline No escolarizados & 26 & 35,62 \\
\hline Absentismo & 17 & 23,29 \\
\hline Conducta escolar inadaptada & 17 & 23,29 \\
\hline Graves dificultades en lectura y escritura & 19 & 26,03 \\
\hline En paro con edad laboral & 19 & 26,03 \\
\hline No participa en activ. formativas y de ocio y t. & 54 & 73,97 \\
\hline Grupo de amigos conflictivo & 54 & 73,97 \\
\hline Ha estado internado en centro de protección & 7 & 9,59 \\
\hline Ha estado internado en centro de reforma & 12 & 16,44 \\
\hline Malos tratos físicos/psíquicos & 9 & 12,33 \\
\hline
\end{tabular}

TABLA 10

En la tabla podemos ver que el mayor porcentaje de características comportamentales asociables al hecho delictivo se halla en los aspectos referentes a las relaciones con iguales ("grupo de amigos conflictivo") y al hecho de la no ocupación del tiempo libre de modo positivo para su desarrollo personal. También aparecen unos porcentajes elevados de menores no escolarizados $(35,62 \%)$ y de menores que consumen sustancias tóxicas $(31,51 \%)$. En este punto conviene matizar que 
el porcentaje real es mucho mayor, pero se trataría básicamente de consumidores de drogas de síntesis (éxtasis, speed, etc.), porros y alcohol, especialmente en fines de semana. Un porcentaje muy bajo estaría referido a heroinómanos y consumidores habituales.

\subsection{Características de la familia al inicio de la medida $(n=73)$}

\begin{tabular}{|l|c|r|}
\hline CARACTERÍSTICAS de la familia al inicio de la medida & N. ${ }^{\circ}$ CASOS & $\%$ \\
\hline Consumo drogas padre/madre & 8 & 10,96 \\
\hline Alcoholismo padre/madre & 7 & 9,59 \\
\hline Encarcelamiento padre/madre & 13 & 17,81 \\
\hline Prostitución manifiesta o encubierta & 2 & 2,74 \\
\hline En paro crónico & 20 & 27,40 \\
\hline En paro temporal & 16 & 21,92 \\
\hline Responsable educativo distinto de padres & 8 & 10,96 \\
\hline Problemas de salud física responsables educativos & 9 & 12,33 \\
\hline Problemas de salud responsables educativos & 10 & 13,70 \\
\hline Problemas de salud física en hermanos & 4 & 5,48 \\
\hline Problemas de salud mental en hermanos & 4 & 5,48 \\
\hline Familia permisiva / Falta de límites & 44 & 60,27 \\
\hline Usuaria habitual de servicios sociales & 31 & 42,47 \\
\hline Problemática delictiva en hermanos & 22 & 30,14 \\
\hline Internamiento de hermanos en centro de reforma & 6 & 8,22 \\
\hline Consumo de alcohol/drogas en hermanos & 19 & 26,03 \\
\hline
\end{tabular}

TABLA 11

Si observamos la tabla y extraemos los datos más significativos, resulta fácilmente deducible el entorno familiar poco normalizado en el que se desenvuelven la mayoría de los menores objeto de esta memoria.

Se trataría, en gran número de casos, de familias permisivas o incoherentes, con ausencia de límites educativos $(60,27 \%)$ y usuarias habituales de servicios sociales $(42,47 \%)$. Además, podemos destacar el porcentaje importante de familias con problemática delictiva en hermanos $(30,14 \%)$ y con una situación cronificada de paro $(27,40 \%)$. Respecto a los aspectos de consumo de alcohol y drogas, las cifras reales siempre son mayores de las que aparecen en la tabla, ya que la recogida de este tipo de datos resulta más complicada, dado que las familias tienden a ocultarlos.

A partir de esta tabla y la anterior podríamos definir el perfil de los menores como: 
- chico (varón),

- entre 14 y 16 años,

- que suele relacionarse con grupo de amigos conflictivo,

- con escasa participación en actividades formativas y de ocio y tiempo libre,

- no escolarizado o con problemática escolar manifiesta (absentismo, conducta desadaptativa, etc.),

- que consume sustancias tóxicas, sobre todo en fines de semana y del tipo de síntesis, "blandas" y alcohol.

Esta descripción, unida a las características de estructuración familiar y vivienda, nos hace pensar que son los valores y actitudes, pautas de conducta habituales en el medio socio-familiar, las circunstancias que mejor explicarían la aparición de las conductas delictivas.

\section{IV.NIVEL DE INTERVENCIÓN (OBJETIVOS).}

De manera global, podríamos afirmar que los objetivos fundamentales o generales del PEI (Programa Educativo Individualizado) que se repiten en cada una de las medidas son:

- Evitar la reincidencia en el menor.

- Lograr la implicación del menor y la familia con el PEI y con el/la educador/a.

\subsection{Libertad vigilada}

TABLA 12

\begin{tabular}{|l|c|c|c|c|}
\hline OBJETIVOS TRABAJADOS EN LIBERTAD VIGILADA & Prop. $^{*}$ & $\%$ & Logr:*: & $\%$ \\
\hline Lograr conocimiento y valoración de sí mismo y de su situación & 76 & 100 & 31 & 62 \\
\hline Adquirir autonomía y responsabilidad & 76 & 100 & 32 & 64 \\
\hline Lograr implicación del menor con el PEI y con el educador & 76 & 100 & 42 & 68 \\
\hline Retomar escolarización & 10 & 13,16 & 3 & 6 \\
\hline Reducir absentismo & 11 & 14,47 & 4 & 8 \\
\hline Aumentar colaboración e implicación en actividades escolares & 19 & 25 & 5 & 10 \\
\hline Obtener certificado de escolaridad & 2 & 2,63 & 1 & 2 \\
\hline Obtener el graduado escolar & 6 & 7,89 & 3 & 6 \\
\hline Ocupar el tiempo libre en una actividad formativa & 38 & 50 & 7 & 14 \\
\hline Adquirir conocimientos y hábitos para el desarrollo de un trabajo & 17 & 22,37 & 4 & 8 \\
\hline Planificar y participar en circuitos laborales adecuados a su capacidad & 19 & 25 & 9 & 18 \\
\hline Reforzar y mejorar actitudes para el mantenimiento del puesto de trabajo & 3 & 3,95 & 2 & 4 \\
\hline Participar en actividades de integración en el barrio & 5 & 6,58 & 3 & 6 \\
\hline Desarrollar una actividad organizada de carácter lúdico-deportivo & 25 & 32,89 & 12 & 24 \\
\hline
\end{tabular}




\begin{tabular}{|l|c|c|c|c|}
\hline Evitar la reincidencia & 76 & 100 & 45 & 90 \\
\hline Lograr que la familia asuma la problemática del menor & 76 & 100 & 31 & 62 \\
\hline Lograr inplicación y colaboración de la familia con el PEl y el/la educador/a & 76 & 100 & 45 & 90 \\
\hline Potenciar actitudes en la familia que mejoren su relación con el menor & 76 & 100 & 32 & 64 \\
\hline Sensibilizar a la familia de la problemática que mejoren su relación con el menor & 76 & 100 & 28 & 56 \\
\hline Ofrecer orientación prelaboral/laboral & 24 & 31,58 & 24 & 48 \\
\hline Adecuar los recursos al interés y las necesidades del menor & 76 & 100 & 42 & 84 \\
\hline Facilitar el acceso a los recursos comunitarios & 76 & 100 & 40 & 80 \\
\hline
\end{tabular}

*Objetivos propuestos: $n=76 . * *$ Objetivos logrados: $n=50$.

En este apartado interesa destacar, como aspectos positivos, la buena implicación de las familias con el PEI y el/la educador/a (90\%), el logro de cambios favorables en la actitud de las familias respecto a los menores $(64 \%)$, la adecuación de los recursos al interés y necesidades del menor (84\%) y la facilitación del acceso a los recursos comunitarios $(80 \%)$.

Como aspectos negativos mencionar que se da un escaso nivel de logro en el objetivo de potenciar la adquisición de hábitos para el desarrollo de un trabajo y también en el objetivo de potenciar actitudes que fomenten la constancia para desarrollar una misma actividad.

\subsection{Prestación de servicios en beneficio de la comunidad}

\begin{tabular}{|c|c|c|c|c|}
\hline TABLA 13 & $(n=10)$ & & $(n=10)$ & \\
\hline OBJETIVOS DE P.S.B.C & Prop. & $\%$ & Logr. & $\%$ \\
\hline Tomar conciencia del daño causado & 9 & 90 & 9 & 90 \\
\hline Participar en la actividad propuesta & 10 & 100 & 10 & 100 \\
\hline Lograr implicación del menor con la actividad y con el/la educador/a & 10 & 100 & 10 & 100 \\
\hline Evitar reincidencia & 10 & 100 & 10 & 100 \\
\hline Lograr implicación de la familia con la actividad y con el/la educador/a & 2 & 20 & 2 & 20 \\
\hline Sensibilizar de la problemálica y necesidades del menor donde se realice la prestación & 10 & 100 & 9 & 90 \\
\hline Ofrecer orientación prelaboral/laboral & 1 & 10 & 1 & 10 \\
\hline
\end{tabular}

\subsection{Internamiento en domicilio durante fines de semana}

Respecto al internamiento en domicilio durante fines de semana, cabe destacar como aspectos positivos la buena implicación tanto del menor como de la familia con el PEI y el/la educador/a. Como aspectos negativos, hay que mencionar la falta de recursos comunitarios para cumplir las horas de prestación de servicios y la dificultad de 


\begin{tabular}{|l|c|c|c|c|} 
TABLA 14 & $(\mathrm{n}=7)$ & \multicolumn{3}{c|}{$(\mathrm{n}=7)$} \\
\begin{tabular}{|l|c|c|c|c|}
\hline OBJETIVOS TRABAJADOS EN INTERNAMIENTO EN DOMICLLIO DURANTE FNES DESEMANA & Prop. & $\%$ & Logr. & $\%$ \\
\hline Lograr conocimiento y valoración de sí mismo y de su situación & 6 & 85,7 & 4 & 57,14 \\
\hline Lograr la implicación del menor con el PEI y con el/la educador/a & 7 & 100 & 6 & 85,71 \\
\hline Orientar al menor en ocupación del tiempo libre y actividades formativas & $\mathrm{I}$ & 14,29 & 1 & 14,29 \\
\hline Permanecer en el domicilio & 7 & 100 & 6 & 85,71 \\
\hline Participar en tareas domésticas & 3 & 42,86 & 3 & 42,86 \\
\hline Evitar reincidencia & 7 & 100 & 7 & 100 \\
\hline Lograr la implicación y colaboración de la familia con el PEl y el/la educador/a & 2 & 28,57 & 1 & 14,29 \\
\hline Facilitar el acceso a los recursos comunitarios & 5 & 71,43 & 4 & 57,14 \\
\hline
\end{tabular}
\end{tabular}

ajustar el horario de tiempo libre del menor al funcionamiento de la entidad prestadora del servicio.

Otro punto importante es que tanto en P.S.B.C. como en internamiento de F.S. el éxito del PEI es mayor debido a que el perfil de familias y de menores es distinto a los de Libertad Vigilada. Se trata de familias y menores bastante normalizados (menores escolarizados, con un ritmo escolar aceptable, familias estructuradas, etc.).

\section{RECURSOS UTILIZADOS}

TABLA 15

\begin{tabular}{|l|r|}
\hline RECURSOS UTILIZADOS & $\mathrm{N}^{\circ}$ \\
\hline Familias acogedoras & 1 \\
\hline Centros escolares & 23 \\
\hline EPAS & 10 \\
\hline Cursos de alfabetización & 6 \\
\hline Módulos de garantía social & 13 \\
\hline Talleres prelaborales & 11 \\
\hline Academias de enseñanza escolar & 1 \\
\hline Academias de F. Profesional & 3 \\
\hline Centros jóvenes & 7 \\
\hline Empleados en puesto laboral & 5 \\
\hline Gimnasios & 11 \\
\hline Asociaciones de ocio y tiempo libre & 2 \\
\hline O.N.Gs & 4 \\
\hline Actividades deportivas organizadas & 15 \\
\hline Empresas de prestación de servicios & 10 \\
\hline
\end{tabular}


El recurso más utilizado es el de centros escolares. Esto es debido a que el perfil de la población estudiada, como ya se ha comentado, responde principalmente a una franja de edad entre 14 y 16 años, edad propia de la última etapa de escolarización obligatoria.

Otro porcentaje importante de recursos utilizados es el correspondiente a la franja de edad de más de 16 años (talleres pre-laborales, EPAs, Módulos de Garantía Social).

En cuanto a la ocupación del Tiempo Libre, la tabla también refleja un porcentaje elevado de recursos utilizados para este fin (Gimnasios, Asociaciones, etc.).

Hay que aclarar que estas cifras corresponden exclusivamente al dato de utilización del recurso, y no al nivel de aprovechamiento del mismo por el menor. Es éste precisamente uno de los problemas fundamentales que encontramos a diario: lograr constancia y continuidad de los menores en las actividades propuestas en el PEI.

Hay que destacar también como recurso de la intervención, aunque no aparecen en la tabla:

- El Equipo de Medio Abierto, como eje principal de la intervención en su labor de apoyo y tutorial.

- Los profesionales de los distintos Centros Sociales del Ayuntamiento.

- Los recursos propios del Centro Social.

- Otros recursos por derivación (p.e. Centros de Salud, etc)

- La propia familia del menor .

\section{VI.VALORACIÓN.}

6.1. Distribución de menores por actitud ante delito, educador y medida (casos de baja) $(n=50)$

Los criterios que hemos seguido para evaluar la actitud del menor ante el delito han sido:

- Positiva (el menor reflexiona adecuadamente sobre las consecuencias del hecho cometido y de su actitud).

- Indiferente (el menor no se plantea las consecuencias de su conducta).

- Negativa (el menor se muestra reforzado en su autoestima por la conducta desarrollada).

Los criterios que hemos tenido en cuenta para evaluar la actitud de la familia ante el delito han sido los siguientes: 
TABLA 16

\begin{tabular}{|l|r|r|r|r|r|r|r|r|r|r|r|r|}
\hline & \multicolumn{4}{|c|}{ MENOR } & \multicolumn{5}{|c|}{ FAMILIA } \\
\cline { 2 - 14 } & + & $\%+$ & $=$ & $\%=$ & - & $\%-$ & + & $\%+$ & $=$ & $\%=$ & - & $\%-$ \\
\hline Actitud ante el delito & 24 & 48 & 21 & 42 & 5 & 10 & 36 & 72 & 11 & 22 & 3 & 6 \\
\hline Actitud ante el educador/a & 38 & 76 & 9 & 18 & 3 & 6 & 37 & 74 & 11 & 22 & 2 & 4 \\
\hline Actitud ante la medida & 28 & 56 & 15 & 30 & 7 & 14 & 36 & 72 & 10 & 20 & 4 & 8 \\
\hline
\end{tabular}

Claves de la tabla anterior: "+" actitud positiva; "=" actitud indiferente; "-" actitud negativa.

- Positiva (la familia apoya al menor en su proceso de reflexión sobre la conducta negativa desarrollada y sus consecuencias).

- Indiferente (la familia no interviene ni apoya al menor en su proceso de reflexión sobre el hecho cometido).

- Negativa (la familia no da importancia al hecho y, de alguna manera, refuerza la conducta delictiva del menor).

Los criterios de evaluación según la actitud del menor ante el educador/a han sido:

- Positiva (el menor acepta al educador/a como figura de referencia).

- Indiferente (No acepta al educador/a como referente educativo, sino como figura coercitiva y de control).

- Negativa (el menor rechaza al educador/a).

Los criterios evaluativos para valorar la actitud de la familia ante el educador/a han sido los siguientes:

- Positiva (la familia acepta el rol del educador/a y colabora).

- Indiferente (la familia no entiende/asume el rol del educador/a).

- Negativa (la familia rechaza la figura del educador/a).

Los criterios que hemos seguido para evaluar la actitud del menor ante la medida (PEI) son:

- Positiva (el menor acepta la medida como una consecuencia válida a su conducta delictiva y colabora activamente en el desarrollo del PEI).

- Indiferente (el menor no entiende/asume la medida como consecuencia al hecho cometido y muestra una actitud pasiva ante el desarrollo del PEI).

- Negativa (el menor rechaza la medida y cualquier actividad que se proponga en el PEI).

Los criterios para evaluar la actitud de la familia ante la medida han sido:

- Positiva (la familia acepta la medida como consecuencia válida a la conducta del menor y se implica en el desarrollo del PEI).

- Indiferente (la familia no asume/entiende la medida y se muestra pasiva ante el PEI). 
- Negativa (la familia rechaza la medida como consecuencia válida a la conducta del menor y no colabora ni se implica en el desarrollo del PEI).

\subsection{Apreciación global del pronóstico de los menores $(n=50)$}

TABLA 17

\begin{tabular}{|l|c|r|}
\hline PRONOSTICO & TOTAL & $\%$ \\
\hline Muy positivo & 10 & 20 \\
\hline Positivo & 14 & 28 \\
\hline Dudoso & 16 & 32 \\
\hline Negativo & 10 & 20 \\
\hline
\end{tabular}

Los criterios que hemos tenido en cuenta en este apartado se han basado en una apreciación global del menor al finalizar la medida y en base a cómo ha desarrollado el P.E.I.

Por ello, consideramos un pronóstico muy positivo de evolución del menor después de la firma del cese de la medida en los casos en los que el menor ha desarrollado un P.E.I. de manera provechosa, cumpliendo la mayoría de los objetivos propuestos, ha aceptado en todo momento la figura del educador/a y la familia es capaz de apoyar y reforzar actitudes positivas en el menor. El pronóstico sería positivo si de los factores mencionados sólo se dan algunos, como, por ejemplo, que el menor haya desarrollado un P.E.I. adecuado, aceptando el rol del educador como referente educativo, aunque por parte de la familia no se haya conseguido un cambio positivo de actitudes respecto al menor. Hemos valorado como pronóstico dudoso los casos en los que el menor ha cumplido una parte del P.E.I. propuesto, pero no se aprecia un cambio real en sus actitudes, dentro de un ambiente familiar permisivo. Por último, hemos considerado un pronóstico negativo en los casos en los que el menor no ha desarrollado un P.E.I. de manera adecuada y desde la familia no hay apoyo suficiente para reforzar actitudes positivas en aquél.

6.3. Nivel de logro de la intervención según criterios de "evitar la reincidencia" y "lograr implicación en las actividades propuestas en el P.E.I."

TABLA 18

\begin{tabular}{|l|c|c|c|}
\hline NIVEL DE LOGRO & LIBERTAD V. & P.S.B.C. & INTERN. F.S. \\
\hline No reincidencia & $90 \%$ & $100 \%$ & $100 \%$ \\
\hline Implicación con el PEI & $68 \%$ & $100 \%$ & $85,71 \%$ \\
\hline
\end{tabular}


Según refleja la tabla, y sobre la base de los criterios que hemos considerado como fundamentales para medir el período de intervención, resulta más que evidente que el nivel de logro alcanzado ha sido muy alto durante el año 96 , lo cual avala considerablemente la validez y eficacia del programa.

\section{VII.COMENTARIO GENERAL Y CONCLUSIONES}

Como primera observación, mencionaremos que en la memoria del año 95 se contabilizaron 63 medidas ejecutadas y 60 menores atendidos, mientras que en la memoria de 1996 se han contabilizado 76 ejecutadas y 73 menores atendidos; por lo tanto, el incremento de un año para otro ha sido de 13 medidas y menores más.

Respecto a las medidas impuestas desde el Juzgado de Menores, la Libertad Vigilada sigue siendo la más utilizada, aunque no debemos olvidar que ha habido un notable aumento de las Prestaciones de Servicios en Beneficio de la Comunidad (de 3 casos en 1995 a 10 en 1996). Sin embargo, no se ha dado un aumento paralelo de entidades colaboradoras para la realización de dichas prestaciones.

Reseñar también que en 1995 se producía una demora de 4 a 5 meses entre el hecho cometido y la fecha de la resolución judicial. En 1996, lejos de acortarse este tiempo, se ha generado un aumento a una media de 6,41 meses entre ambas circunstancias, con lo que supone de pérdida de efectividad educativa con vistas a plantear al menor un proyecto socializador basado primariamente en la responsabilidad del hecho cometido. El tiempo transcurrido entre el hecho cometido, la resolución judicial y el inicio de la ejecución de la medida debería ser el menor posible dada la importancia educativa que posee para el menor la inmediata conexión entre su conducta delictiva y la medida que se desprende de la resolución.

El perfil del menor infractor y el de la familia de éste sería el de un chico de unos 15 años de edad, sin escolarizar, con grupo de amigos conflictivo, que consume sustancias tóxicas de las que se podrían calificar como "blandas" y de "síntesis" (sobre todo, en fines de semana), que convive en un núcleo familiar de pareja relativamente estable, con situaciones de paro crónico y nivel económico, por tanto, más bien bajo, usuarias habituales de servicios sociales y con hermanos que consumen también sustancias tóxicas y han manifestado conductas delictivas (cfr. Distinta tipología de familia según el tipo de medida).

El EMA (educador de medio abierto) sigue siendo la figura relevante en la intervención con el menor y, en gran número de casos, el 
único recurso educativo válido, ejerciendo funciones de tutoría social. Esto se da, sobre todo, en los casos que llegan durante el primer semestre del año, debido a que los talleres pre-laborales y demás cursos de formación general ya están iniciados desde el último trimestre del año anterior.

Resaltar también la dificultad de encontrar recursos adecuados para chicos menores de 16 años, para los que la escuela no es ya un recurso válido y tampoco pueden acceder a talleres pre-laborales ni al mercado laboral reglado por falta de edad. Todo esto nos puede conducir al riesgo de agotamiento del EMA como único recurso, especialmente, en los casos en los que la libertad vigilada se prolonga más allá de 8 ó 9 meses. En tales casos, se cuestionaría la efectividad y eficacia de las medidas de libertad vigilada de larga duración, ya que resulta complicado mantener el interés del menor dada la situación de escasez de recursos anteriormente descrita.

Cada vez es mas frecuente la necesaria coordinación con distintas entidades con las cuales tiene relación el educador debido a la concreción del PEI de cada menor (cfr. ver tabla de recursos utilizados.) Especial mención merece la relación con los profesionales de los distintos centros sociales del Excmo. Ayuntamiento de Alicante, donde el trabajo conjunto es bastante satisfactorio y la utilización de servicios gradualmente ira a más (sesiones terapéuticas y diagnósticas con el psicólogo/a, tratamientos de toxicomanías, ayudas puntuales económicas, intervenciones familiares, etc.)

El programa de seguimiento de medidas judiciales de medio abierto, se convierte en un buen catalizador de «destape» de necesidades (tanto del propio menor como de su entorno) que conlleva al incremento del nivel de intervención. En el caso del menor, es el EMA el profesional indicado para dar respuesta a la problemática presentada. En el caso de la familia, el EMA derivará al centro social la problemática para su conocimiento y actuación.

Es preocupante el incremento del numero de menores con problemática de toxicomanías. Este es un tema que no habrá que perder de vista en su evolución de cara a una intervención específica.

Otro aspecto que se hace cada vez mas necesario, en la intervención con el menor, es la necesaria implicación de la familia en el proceso resocializador. Implicar a la familia en tal proceso supone trabajar con ella y, en muchos casos, descubrimos otras carencias sobre las que hay que actuar.

Al finalizar el año, desde la Dirección General de Asuntos Sociales, se ha planteado un cambio en el procedimiento de derivación de los casos por parte de la Dirección Territorial. Sería deseable que una 
vez adaptado el funcionamiento no se produzcan desfases en la formalización del acta de inicio de la medida, estando presentes el menor y sus responsables educativos.

El programa de Seguimiento de Medidas Judiciales en Medio Abierto, según los datos aportados, ofrece una respuesta bastante apropiada a la realidad de los menores infractores (cfr. ver tabla de nivel de logro de la intervención) que otras actuaciones (internamientos cerrados) menos económicas y, en muchos casos, no mas idóneas para los objetivos pretendidos (responsabilización, no reincidencia, inserción, normalización social del menor).

Queda pendiente la profundización en los distintos temas mencionados (red de recursos, intervención familiar mas específica/especializada, etc.).

\section{PERSPECTIVAS PARA 1997}

I. Continuar con un sistema de evaluación cada vez más preciso, utilizando una base de datos adaptada y mejorada, en base a la hasta ahora utilizada.

II. Según el tipo de medidas ejecutadas en la actualidad, existe una serie de recursos que se han comprobado como válidos, pero insuficientes en bastantes casos. Seguiremos utilizando la oferta publica de los mismos, trabajando una adaptación mejor de estos a lo largo del año, así como sugeriremos otros recursos posibles que favorezcan la inserción social de los menores. Así mismo, según la frecuencia de las medidas, habría que trabajar más para la búsqueda de entidades colaboradoras para llevar a cabo la medida de PSBC.

III.Desarrollar un programa de mediación, dentro del programa general, para menores infractores en colaboración con el Equipo Técnico del Juzgado de Menores y con el/la Juez/a de menores.

IV.Desarrollar un proyecto de intervención grupal (en grupos pequeños) que complemente el trabajo eminentemente individual del programa, según características y necesidades de los menores, incidiendo en temas de comunicación, habilidades sociales, competencia social, resolución de conflictos, etc. que resulta necesario para muchos de estos menores.

V. Establecer un mecanismo fluido entre las distintas instancias que intervienen con el menor (Servicios Sociales Autonómicos, Juzgado de Menores, Ayuntamiento), así como precisar el procedimiento administrativo y funcional para llevar a efecto las medidas (comunicación de la resolución, informes del Equipo Técnico del Juzgado, citaciones, plazos, etc.). 